\title{
ANOMALÍAS DENTARIAS DE NÚMERO, EVALUADAS EN TOMOGRAFÍAS COMPUTARIZADAS DE HAZ CÓNICO.
}

\author{
DENTAL NUMBER ABNORMALITIES, EVALUATED IN COMPUTED \\ TOMOGRAPHIES CONE BEAM.
}

\author{
Kevin Roberto Romero Diaz ${ }^{1}$, Ana Paola Trevejo Bocanegra ${ }^{2}$ \\ ${ }^{1}$ Facultad de Odontología, Universidad de Guayaquil. Guayaquil, Ecuador. \\ ${ }^{2}$ Facultad de Estomatología Roberto Beltrán, Universidad Peruana Cayetano Heredia. Lima, Perú.
}

\begin{abstract}
Resumen
Objetivos: el objetivo del presente fue determinar la frecuencia de anomalías dentarias de número del servicio de radiología de la facultad de estomatología Universidad Peruana Cayetano Heredia, Lima en el periodo 2017-2018. Materiales y métodos: Se evaluaron 1183 tomografías de haz cónico (TCHC), y se determinó la frecuencia de agenesias dentarias (AD) según grupo etario, sexo y localización. Dientes supernumerarios (DS) según grupo etario, sexo, localización, alteraciones de forma, tamaño, posición y erupción. Resultados: Se hallaron anomalías dentarias de número en 108 TCHC, se obtuvo $93.52 \%$ de casos con DS y $6.48 \%$ de AD. Los DS se presentan con mayor frecuencia de 6 a 15 años con $51.49 \%$, más en hombres con $53.47 \%$, localización más frecuente la zona anterior $52.48 \%$, la forma cónica con $30.69 \%$, dientes con microdoncia, el $24.75 \%$ en posición horizontal, por último $74.26 \%$ de DS que estaban incluidos. Las AD se presentaron en pacientes de 7 a 14 años más en hombres $85.71 \%$ y en el sector anterior. Conclusiones: El $9 \%$ de la población se encontraron con anomalías de número. La localización más frecuente fue la zona anterior de la arcada. Además, no se presentó diferencia significativa de AD y DS entre hombres y mujeres.
\end{abstract}

Palabras clave: anomalías dentarias, supernumerario, agenesia dentaria, tomografía computarizada de haz cónico. (DeCS).

\begin{abstract}
Aim: the objective of this was to determine the frequency of dental anomalies of the radiology service number of the stomatology faculty Universidad Peruana Cayetano Heredia, Lima in the period 2017-2018. Material and methods: 1183 cone beam computed tomography (CBCT) were evaluated, and the frequency of dental agenesis (DA) was determined according to age group, sex and location. Supernumerary teeth (ST) according to age group, sex, location, shape changes, size, position and eruption. Results: Dental anomalies of number were found in 108 CBCT, 93.52\% of cases with ST and $6.48 \%$ of DA were obtained. DS occur more frequently from 6 to 15 years with 51.49\%, more in men with 53.47\%, more frequent location in the anterior area $52.48 \%$, the conical shape with $30.69 \%$, teeth with microdontia, $24.75 \%$ in a horizontal position, finally $74.26 \%$ of ST that were included. DA occurred in patients 7 to 14 years older than in men $85.71 \%$ and in the anterior sector. Conclusions: $9 \%$ of the population found number abnormalities. The most frequent location was the anterior area of the arch. Furthermore, there was no significant difference between DA and ST between men and women.
\end{abstract}

Key words: dental anomalies, supernumerary tooth, dental agenesis, cone beam computed tomography. (MeSH).

\section{Introducción}

Las anomalías son un cambio o diferencia respecto de lo que es normal o natural. Las anomalías dentarias de forma, tamaño, número, posición y erupción pueden presentarse en denticiones tanto permanente como en la decidua. Si bien es cierto que su etiología no es tan clara, existe evidencia que atribuyen a los genes como determinantes para la presencia de anomalías de número: agenesias dentarias (AD) o anodoncia e hiperodoncia o dientes supernumerarios (DS). ${ }^{1}$

Los DS son dientes extras que erupcionan en las arcadas dentarias. Éstos pueden clasificarse según su morfología 
en: eumórficos si muestran características normales o dismórficos si muestran anormalidades en cuanto a su forma. En cuanto a su posición dentro del maxilar son: mesiodens, paramolar y distomolar. La hiperodoncia suele ser ocasional, pero existen componentes hereditarios pueden afectar esta condición. Por otro lado, pueden estar asociados a síndromes como el Síndrome de Gardner o la Displasia Cleidocraneal entre otros. Los DS, pueden estar relacionados en el retardo de la erupción de otras piezas, impactación, desplazamiento o persistencia de piezas deciduas, generando maloclusiones dentarias que repercuten la función, estética y autoestima. ${ }^{2}$

Los DS pueden presentar alteración de tamaño como lo son la macrodoncia y la microdoncia, siendo esta última la más frecuente. La macrodoncia es el aumento del tamaño del diente; se puede mostrar en una pieza o en varias, con más frecuencia en los incisivos centrales superiores de la dentición permanente y en caninos permanentes. La microdoncia es todo lo contrario, es el tamaño reducido de la corona o únicamente de la raíz. Se relaciona a trisomía del cromosoma 21 y Displasia Ectodérmica. Si no existe alteración sistémica, su predilección suelen ser en incisivos laterales superiores de la dentición permanente; así mismo, algunas de éstas presentan modificación en la morfología. ${ }^{3}$

La mayoría de los DS tienen alteración en su posición, estos pueden ser normales, invertidos, horizontales o transversales orientándose la corona hacia una de las tablas óseas. Su forma también presenta alteraciones entre estos se encuentran los dientes en forma de clavija son dientes donde el ancho del diente es mayor a nivel cervical que incisal, dando lugar a una inversión de los diámetros mesiodistales del diente. ${ }^{4}$

Los DS pueden interferir con la erupción ya que estos tienen tendencia a impactarse siendo una barrera mecánica que ocasiona la interrupción de la erupción por la vía normal. Cuando no existe esta barrera mecánica en la erupción se designa como retención. Otra variación en donde la pieza dentaria erupciona en una posición fuera de lo normal pero dentro de su arcada dentaria sin afectar dientes vecinos se la denomina una erupción ectópica. ${ }^{5}$

Los DS suelen llevar una posición ectópica o una trayectoria anormal comparada al de otros dientes. El diagnóstico temprano es el paso más importante para prevenir posibles complicaciones, estas pueden ser diastemas, desplazamientos de estructuras de importancia, rotaciones, impactaciones dentarias, lesiones quísticas y reabsorción de dientes adyacentes. Si bien la etiología de este fenómeno es desconocida, existen tres teorías que intentan explicar la formación de estos. La primera es la teoría dicotómica según la cual hay una división en el estadio de brote del diente, que va a conllevar a dos o más gérmenes dentarios por separado. La segunda teoría y la más aceptada es la hiperactividad de las células de la lámina dental, donde induce la diferenciación de ésta en otro brote de diente adicional que resulta en un DS. La tercera teoría es la combinación de factores ambientales o genéticos y también se encuentra relacionada con distintos síndromes, uno de ellos la Displasia Cleidocraneal.6,7

La TCHC está fundamentalmente diseñada para producir imágenes de alta resolución e información tridimensional para aplicaciones dentales. Gracias a los avances tecnológicos tiene la ventaja de ser más precisa en el diagnóstico. En la TCHC los vóxeles son isotrópicos (iguales en longitud, altura y profundidad), lo que lleva a obtener mediciones geométricamente precisas en cualquier plano.

La gran mayoría de los estudios sobre anomalías dentarias se han realizado usando la radiografía panorámica, como una radiografía de rutina. Sin embargo, son pocos estudios que usan a las tomografías computarizadas de haz cónico como herramienta diagnóstica, y la mayoría de ellos son en inglés. Tomando en consideración lo expresado, el presente estudio tuvo como objetivo determinar la frecuencia de anomalías dentarias de número, evaluadas en TCHC, del servicio de radiología de la facultad de estomatología Universidad Peruana Cayetano Heredia, Lima 2017-2018.

\section{Materiales y métodos}

La población estuvo compuesta por $1183 \mathrm{TCHC}$ de pacientes que acudieron al Servicio de Radiología Oral y Maxilofacial realizadas en el Centro Dental Docente de la Universidad Peruana Cayetano Heredia en la Sede San Martin de Porres en el periodo 2017-2018 Se aplicaron los criterios de selección como: TCHC con error de movimiento que no permita la visualización correcta de las piezas dentarias y TCHC de pacientes con sospecha de un síndrome o alteración sistémica. Por lo cual se trabajó con 108 volúmenes tomográficos.

Por cada TCHC se recolectaron datos demográficos como el numero de DS, sexo y edad, después se analizaron las características de los DS como su localización, forma, tamaño, posición y erupción.

Los DS se clasificaron según su forma en cónico, barril y mora. Según su tamaño en macrodoncia y microdoncia. Según su posición en horizontal, invertida y transversal y por último según su erupción incluido e impactado.

La observación de cada pieza dentaria se hizo siguiendo el orden del primer al cuarto cuadrante. En la ficha de recolección de datos las $\mathrm{AD}$ solo se pudieron comprobar en dentición mixta. Se utilizó el método de observación directa 


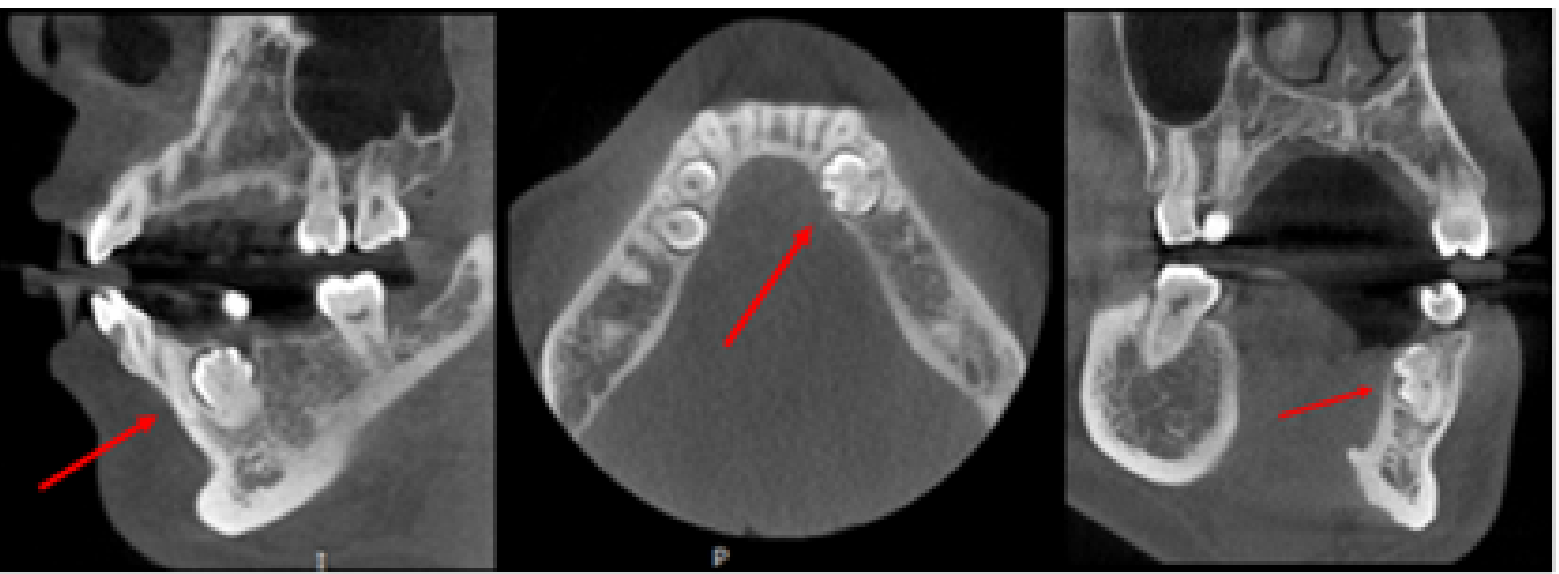

Fig. 1. Ejemplo representativo de una tomografía computada de haz cónico (TCHC) en cortes sagital, axial y coronal respectivamente y muestra un diente supernumerario con forma de mora en próximo contacto con el canino, primer y segundo premolar del maxilar inferior.

en donde se analizó cada volumen de TCHC adquirido con el equipo modelo CS 9300 de la marca Carestream® (filiación), por medio del software visualizador CS 3D Imaging $®$, en una pantalla de 16 pulgadas de marca Lenovo® de resolución 1600 x 900. Se anotaron las observaciones específicas para cada una de las variables consignadas en la investigación en una ficha de recolección de datos en Excel 2010 elaborado para este propósito.

\section{Resultados}

Se revisaron $108 \mathrm{TCHC}$ de las cuales la distribución de genero fue $48(44.45 \%)$ mujeres y $60(55.55 \%)$ hombres. En lo que corresponde las alteraciones dentarias de número se encontraron $\mathrm{SN}=101(93.52 \%)$ y $\mathrm{AD}=7(6.48 \%)$.

\section{Frecuencia de las alteraciones dentarias de número}

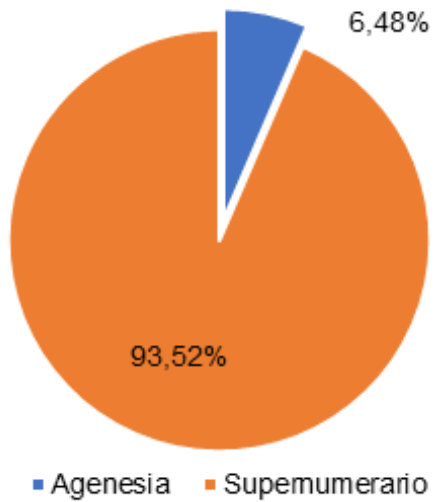

Fig. 2. Frecuencia de las alteraciones dentarias de número.

\section{Discusión}

En la búsqueda de evitar posibles complicaciones como diastemas, desplazamiento de estructuras, apiñamiento o incluso rotaciones e impactaciones de los dientes, se recomienda diagnóstico temprano de los DS, y de mucha mas efectividad si se lo hace con una TCHC que nos brinda datos mas reales y precisos a diferencia de una radiografía en $2 \mathrm{D}$.

Según los resultados de este estudio el porcentaje de dientes DS es de $93.52 \%$ siendo superior al de la AD que presentó un $6.48 \%$, estos resultados difieren con los valores de Mc Kibben y Brearley ${ }^{8}$ donde la $\mathrm{AD}$ presenta un porcentaje mayor $(74.55 \%)$ que los DS $(25.45 \%)$.

En cuanto al grupo etario nuestro estudio difiere con los resultados de Leco Berrocal, ${ }^{9}$ donde presenta mayor porcentaje de dientes supernumerarios entre edad entre 21 y 30 años $(47.6 \%)$ a diferencia de nuestros resultados en donde pacientes de 6 a 15 años presentaron mayor incidencia $(51.49 \%)$. Esta observación puede deberse a que gran porcentaje de DS tienden a ser un hallazgo radiográfico en ese rango de edad, y a la baja población pediátrica de dicho estudio. En el estudio de Salcido-García JF ${ }^{10}$ los resultados fueron similares a los nuestros, en el rango de edad de 4 a 24 años presentaba $44.4 \%$ de presencia de DS.

El presente estudio confirma el dimorfismo asociado al sexo de DS; ya que la incidencia es mayor en hombres, lo cual se corrobora con varios estudios como el de Leco Berrocal $^{9}$ con $71.4 \%$ en hombres versus $28.6 \%$ en mujeres, con esto se confirma la mayor tendencia de DS en hombres.

En el estudio de Jammal Dobles ${ }^{11}$ se informa la presencia de un mayor número de mesiodens con un 


\begin{tabular}{|c|c|c|c|}
\hline & & $n$ & $\%$ \\
\hline \multicolumn{4}{|l|}{ Grupo Etario } \\
\hline & 6 a 12 años & 52 & 51.49 \\
\hline & 13 a 19 años & 28 & 27.72 \\
\hline & 20 a 40 años & 14 & 13.86 \\
\hline & 41 a 60 años & 5 & 4.95 \\
\hline & $\begin{array}{ll}61 & \text { en } \\
\text { adelante } & \end{array}$ & 2 & 1.98 \\
\hline \multicolumn{4}{|l|}{ Sexo } \\
\hline & Masculino & 54 & 53.47 \\
\hline & Femenino & 47 & 46.53 \\
\hline \multicolumn{4}{|l|}{ Localización } \\
\hline & Mesiodens & 53 & 52.48 \\
\hline & Parapremolar & 45 & 44.55 \\
\hline & Paramolar & 2 & 1.98 \\
\hline & Distomolar & 1 & 0.99 \\
\hline \multicolumn{4}{|l|}{ Forma } \\
\hline & No Presenta & 62 & 61.39 \\
\hline & Cónico & 31 & 30.69 \\
\hline & Barril & 1 & 0.99 \\
\hline & Mora & 7 & 6.93 \\
\hline
\end{tabular}

Tamaño

$\begin{array}{lll}\text { No Presenta } & 70 & 69.31 \\ \text { Microdoncia } & 31 & 30.69 \\ \text { Macrodoncia } & 0 & 0.00\end{array}$

Posición

$\begin{array}{lll}\text { No Presenta } & 51 & 50.5 \\ \text { Horizontal } & 25 & 24.75 \\ \text { Invertida } & 18 & 17.82 \\ \text { Transversal } & 7 & 6.93\end{array}$

Tabla 1. Frecuencia de grupo etario, sexo, localización, alteraciones de forma, tamaño, posición y erupción en dientes supernumerarios.

\begin{tabular}{llll}
\hline & & $\mathbf{n}$ & $\%$ \\
\hline \multirow{4}{*}{ Grupo Etario } & & \\
& 6 a 12 años & 5 & 71.4 \\
\multirow{4}{*}{ Sexo } & 13+ años & 2 & 28.6 \\
& Masculino & 6 & 85.71 \\
\multirow{4}{*}{ Localización Zona Anterior } & 6 & 85.71 \\
& Femenino & 1 & 14.29 \\
& Zona Premolares & 1 & 14.29 \\
& Zona Molares & 0 & 0 \\
\hline
\end{tabular}

Tabla 2. Frecuencia de grupo etario, sexo y localización en agenesias dentarias.
$35 \%$, este resultado coincide con la presente investigación donde se evidenció mayor presencia de mesiodens con un $52.48 \%$. Salcido- García JF ${ }^{10}$ reportó en segundo lugar a los parapremolares y por último a los molares. La presente investigación sugiere la hipótesis de que, según la cronología dentaria de dientes deciduos y permanentes, los incisivos son los primeros en formarse y erupcionar por ende se presume que las alteraciones se manifestaran con mayor tendencia en esa región en específico.

En la presente investigación, la gran mayoría de dientes supernumerarios no presenta alteración en su forma, sin embargo, la alteración con más prevalencia que se presentó fue la forma cónica con un $30.69 \%$. Estos resultados coinciden con los de Jammal N. ${ }^{11}$ donde encontró un $20 \%$ de dientes de forma cónica y con los de Kara M. ${ }^{12}$ donde se encontró un $25.3 \%$ de DS con dicha alteración en la forma. Conociendo estos datos nos surge la hipótesis de que el germen dentario al estar en un sector que no le corresponde no se desarrolla de manera correcta en la arcada por eso es probable que adquiera esa alteración en la forma.

Según el tamaño, los dientes supernumerarios pueden presentar macrodoncia, microdoncia o no tener ninguna alteración. Los resultados de la presente investigación indican que la gran mayoría no presenta alteración en el tamaño; sin embargo, se pudo evidenciar que el $30.69 \%$ de DS presentaba microdoncia.

En cuanto a la posición de los DS, la presente investigación dio como resultado que el $50.5 \%$ de estos no presentaba una alteración y la posición horizontal fue la siguiente en aparecer con $24.75 \%$, difiriendo con Yun-Hoa Jung ${ }^{13}$ que encontró en su estudio $21.2 \%$ en posición normal y $60.6 \%$ de DS en posición invertida en una población coreana. Nuestros resultados coinciden con los de Kara M. ${ }^{12}$ que encontró $52.1 \%$ de DS en posición normal.

En una población india, Khandelwal ${ }^{14}$ informó que el $57.3 \%$ de los DS estaban erupcionados y el $42.68 \%$ no estaban erupcionados, distinto a nuestros resultados donde solo el $21.78 \%$ estaban erupcionados. Syriac ${ }^{15}$ en su estudio menciona que el $34.3 \%$ de los DS encontrados estaban impactados o incluidos, a diferencia de nuestro estudio que se encontraron $74.26 \%$ incluidos mientras que el $3.96 \%$ se encontraban impactados. Estos resultados podrán variar dependiendo de la posición y forma del diente, además del espacio requerido para su erupción.

Con respecto a los resultados de $\mathrm{AD}$ recolectados, en el grupo etario de 6 a 12 años y más de 13 años se encontraron del total de anomalías de numero un $6.48 \%$, de los cuales el $85.71 \%$ de estos se encontró en el sexo masculino, difiriendo con el estudio de Gokkaya ${ }^{16}$ donde encontró mayor disposición en el sexo femenino. Además, manifestó encontrar casos de AD más frecuentes en pacientes de 10 a 12 años de edad, 


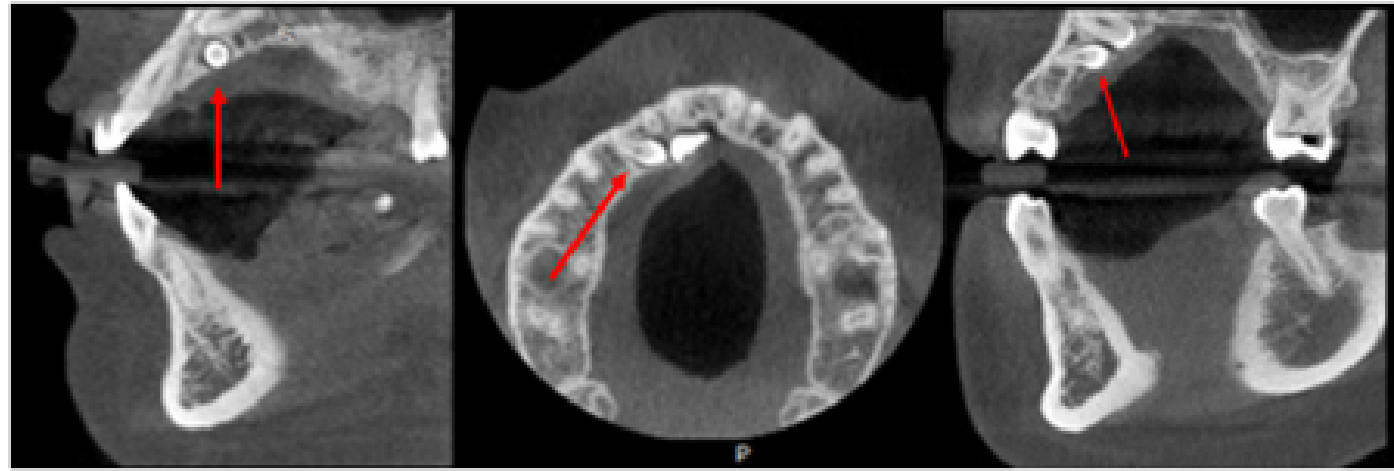

Fig. 3. Ejemplo representativo de una tomografía computada de haz cónico (TCHC) en cortes sagital, axial y coronal respectivamente y muestran un diente supernumerario con microdoncia en posición invertida en próximo.

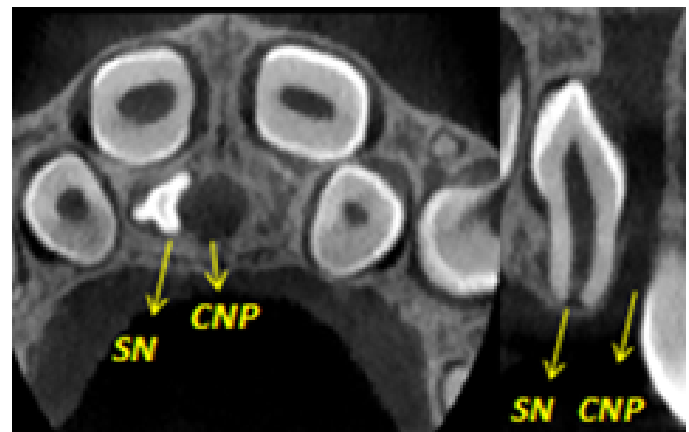

Fig. 4. Ejemplo representativo de una tomografía computada de haz cónico (TCHC) en corte axial y coronal que muestra un diente $(\mathrm{SN})$ en el maxilar superior en posición invertida próximo al conducto naso palatino (CNP)contacto con el central del maxilar superior.canino, primer y segundo premolar del maxilar inferior.

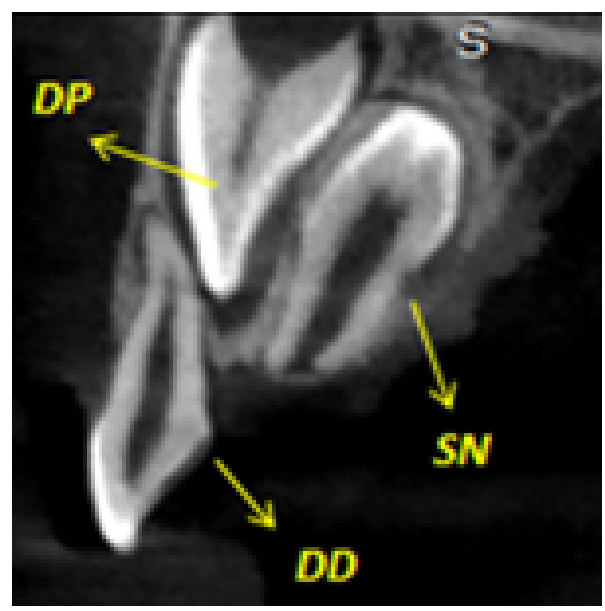

Fig. 5. Ejemplo representativo de una tomografía computada de haz cónico (TCHC) que muestra en un corte sagital un diente supernumerario (SN) con alteración en la forma en el maxilar superior en posición invertida que dificulta la correcta erupción. y cuya localización más frecuente es el lado izquierdo de la mandíbula.

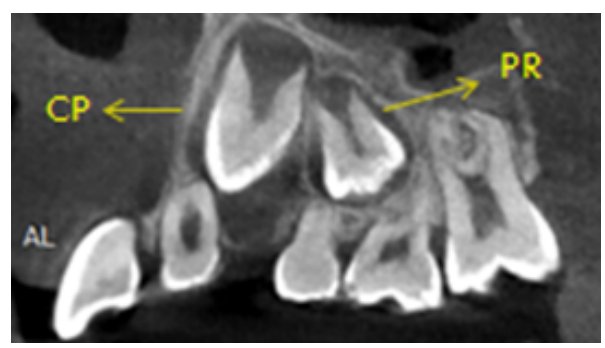

Fig. 6. Ejemplo representativo de una tomografía computada de haz cónico (TCHC) que muestra en un corte sagital al canino permanente $(\mathrm{CP})$, al primer premolar (PR) y la agenesia dentaria (AD) del segundo premolar. del diente permanente (DP), el diente deciduo (DD) no se encuentra afectado.(CNP)contacto con el central del maxilar superior.canino, primer y segundo premolar del maxilar.

Las limitaciones del presente estudio fueron no contar con una base de datos más grande y no haber considerado en que maxilares (superior o inferior) se presentan los DS y las AD.

En base a los resultados encontrados y su comparación con estudios de DS se recomienda investigar de una manera más profunda las causas de mayor incidencia de mesiodents, predisposición de sexo, causas de mayor incidencia de microdoncia, al igual de su alteración de forma cónica y su relación con los odontomas. En cuanto a AD se recomienda investigar si está relacionada con el atraso de la erupción dentaria ya que se encontraron casos fuera de la cronología de erupción

\section{Conclusiones}

Dentro de este estudio podemos destacar que el sexo masculino con un rango de edad de 0-3 años, tuvo mayor participación Las anomalías de número siempre estarán presentes en una población a pesar de su baja frecuencia. 
La localización más frecuente fue la zona anterior de los maxilares. La AD y los DS fueron mayores en hombres, pero estas pueden variar según la población. El conocimiento de la edad, sexo, prevalencia en maxilar y un correcto examen imagenológico ayudará al odontólogo a realizar un mejor diagnóstico. Los DS pueden estar en contacto cercano con el hueso cortical del piso de fosas nasales, el conducto nasopalatino, el conducto dentario inferior y las raíces de uno de los dientes adyacentes. Por lo tanto, un análisis imagenológico 3D con TCHC es importante para 12 la localización exacta y la planificación del tratamiento, incluida la extracción quirúrgica.

Conflicto de intereses: Los autores del presente estudio 13 manifiestan que no existe ningún conflicto de intereses en relación al tema de estudio.

\section{Referencias}

1 Demiriz L, Hazar Bodrumlu E, İçen M, Durmuşlar MC. Evaluation of the accuracy of cone beam computed tomography on measuring impacted supernumerary teeth. Scanning. 2016; 38(6):579-84.

2 Garib DG, Peck S, Gomes SC. Increased occurrence of dental anomalies in patients with second premolar agenesis. Angle Orthod. 2009; 79(3):436- 41.

3 Kathariya MD, Nikam AP, Chopra K, Patil NN, Raheja H, Kathariya R. Prevalence of Dental Anomalies among School Going Children in india. J Int Oral Health. 2013; 5(5):10-4

4 Martín-González J, Sánchez-Domínguez B, TarilonteDelgado ML, Castellanos-Cosano L, Llamas-Carreras JM, López-Frías FJ, et al. Anomalías y displasias dentarias de origen genético-hereditario. Av Odontoestomatol. 2012; 28(6):287-301.

5 Gamba GD, Maria AB, Vellini FF, Okada OT. Associated dental anomalies: The orthodontist decoding the genetics which regulates the dental development disturbances. Dental Press J Orthod. 2010; 15 (2): 138-53.

6 Albert A, Mupparapu M. Cone beam computed tomography review and classification of mesiodens: Report of a case in the nasal fossa and nasal septum. Quintessence Int. 2018; 49(5):413-7.

7 Klein OD, Oberoi S, Huysseune A, Hovorakova M, Peterka M, Peterkova R. Developmental disorders of the dentition: an update. Am J Med Genet C Semin Med Genet. 2013; 163C (4):318-32.

8 Alcántara Mena C. Prevalencia y distribución de agenesias dentarias y dientes supernumerarios en pacientes de 7 a 18 años de edad atendidos en el Centro Médico Naval. Enero 2003 - Julio 2004. Lima, Peru; 2005: 1-61.

9 Leco Berrocal MI, Martín Morales JF, Martínez González JM. An observational study of the frequency of supernume- rary teeth in a population of 2000 patients. Med Oral Patol Oral Cir Bucal. 2007; 12: E134-8.

10 Salcido-García JF, Ledesma-Montes C, Hernández-Flores F, Pérez D, Garcés-Ortíz M. Frequency of supernumerary teeth in Mexican population. Med Oral Patol Oral Cir Bucal. 2004; 9:407-9.

1 Jammal N, Silva R. Prevalencia de dientes supernumerarios en una muestra mexicana. Rev Mex Ortod. 2015; 3(2):88-91.

12 Kara, M. İ., Aktan, A. M., Ay, S., Bereket, C., Şener, İ., Bülbül, M., Polat, H. B. Characteristics of 351 supernumerary molar teeth in Turkish population. Medicina oral, patología oral y cirugía bucal 2011, 17(3), E395-400.

13 Jung, Y. H., Kim, J. Y., \& Cho, B. H. The effects of impacted premaxillary supernumerary teeth on permanent incisors. Imaging science in dentistry 2016, 46(4), 251-8.

14 Khandelwal P, Rai AB, Bulgannawar B, Hajira N, Masih A, Jyani A. Prevalence, Characteristics, and Morphology of Supernumerary Teeth among Patients Visiting a Dental Institution in Rajasthan. Contemp Clin Dent. 2018; 9(3):34956.

15 Syriac G, Joseph E, Rupesh S, Philip J, Cherian SA, Mathew J. Prevalence, Characteristics, and Complications of Supernumerary Teeth in Nonsyndromic Pediatric Population of South India: A Clinical and Radiographic Study. J PharmBioallied Sci. 2017; 9(Suppl 1): S231-6.

16 Gokkaya, B., \& Kargul, B. Prevalence and Pattern of NonSyndromic Hypodontia in a Group of Turkish Children. Acta Stoma. Croatica 2016, 50(1), 58-64.

Recibido: 20 de septiembre de 2020 Aceptado: 29 de abril de 2021 